\title{
MiR-217 Promotes Osteogenic Differentiation of Periodontal Ligament Stem Cells Though EZH2- mediated Epigenetically Regulating Wnt Pathway
}

Hao Jiang ( $\nabla$ jianghao20083030@163.com )

Department of Stomatology, the Fifth Central Hospital of Tianjin, Tianjin 300450, China https://orcid.org/0000-0003-0640-9416

Peizeng Jia

Peking University School of Stomatology

Research article

Keywords: MiR-217, EZH2, osteogenic differentiation, PDLSCs

Posted Date: August 26th, 2020

DOI: https://doi.org/10.21203/rs.3.rs-64422/v1

License: (c) This work is licensed under a Creative Commons Attribution 4.0 International License.

Read Full License 


\section{Abstract}

Background: This study aimed to investigate the potential roles of miR-217 and EZH2 in the osteogenic differentiation of PDLSCs.

Methods: The expression pattern of miR-217 and EZH2 in PDLSCs during osteogenesis was detected by qRT-PCR. Then gain-and loss-of-function was performed to confirm the roles of miR-217 and EZH2 in the osteogenic differentiation of PDLSCs. Alkaline phosphatase and alizarin red S staining were used to detect the activity of osteoblasts and mineral deposition. Western blot and qRT-PCR were performed to detected osteogenic markers to conform osteogenesis phenotype. Furthermore, dual luciferase reporter assay was conducted to analyze the binding of miR-217 to EZH2. Chromatin immunoprecipitation analysis were performed to explore the mechanism of EZH2 and miR-217 on Wnt pathway.

Results: miR-217 was significantly upregulated during the osteogenic differentiation, whereas EZH2 was significantly downregulated. Moreover, knockdown of miR-217 and overexpression of EZH2 inhibited the ALP activity, ARS staining, and expression of osteogenic genes. Furthermore, overexpression of EZH2 partially reversed the effects of miR-217 overexpression on osteoblast differentiation. Finally, miR-217 overexpression promoted the expression of Wnt genes, resulting in the activation of the Wnt/ $\beta$-catenin signaling pathway by targeting EZH2.

Conclusion: Our results demonstrated that miR-217 is induced by osteogenic stimuli and promotes osteogenic differentiation partly by targeting the $\mathrm{EZH} 2 / \mathrm{Wnt} 1 / \beta$-Catenin signaling pathway. This study provides a novel understanding of the mechanisms of osteogenic differentiation, and suggests a potential method for promoting bone formation.

\section{Introduction}

Periodontitis is a infectious chronic inflammatory disease, which could enhance progressive damage to periodontal support tissue and result in tooth loss in adults(Joshi et al., 2016). Periodontal ligament stem cells (PDLSCs), possessing a high proliferative, self-renewal and multi-differentiation abilities features, has been reported to be applied to restoration and regeneration(Son et al., 2019, Nagata et al., 2017, Menicanin et al., 2014). Treatment using PDLSCs could restore the damaged or diseased periodontal tissues. Hence, better understanding of the potential mechanisms on PDLSCs differentiation could be helpful for the treatment of periodontitis.

MicroRNAs (miRNAs), small non-coding RNAs consisting of approximately 21-23 nucleotides, plays an important role in regulating gene expression, cell cycle, and cell differentiation(Xu et al., 2019). During stem cell differentiation, large amounts of miRNAs have been reported to execute its functions in in mesenchymal stem cell osteogenesis(Eskildsen et al., 2011). For instance, miR-138(Eskildsen et al., 2011), miR-132(Xu et al., 2019) and miR-23a(Zhang et al., 2019) were reported to regulate osteogenesis of mesenchymal stem cells. MiR-223, miR-146a, miR-203, miR-155 and miR-142 were considered as potential biomarkers for periodontal disease(Schmalz et al., 2016). MiR-217 was involved in multiple 
diseases, such as chronic heart failure(Nie et al., 2018), membranous nephropathy(Li et al., 2017), neuropathic pain(Jiang et al., 2019) and malignant tumor(Zhu et al., 2016, Nishioka et al., 2014, Zhao et al., 2010). However, the role of miR-217 on the osteogenesis differentiation of PDLSCs is unknown.

In this study, we firstly reported that miR-217 is highly expressed in PDLSCs after osteogenic induction. We also found that miR-217 promoted the osteogenesis differentiation of PDLSCs through targeting the $3^{\prime} U T R$ of EZH2, resulting in activating Wnt/ $\beta$-catenin signaling pathway.

\section{Materials And Methods}

\section{Isolation and primary culture of PDLSCs}

Healthy premolars were collected from four donors who underwent extractions for orthodontic reasons. This study was approved by the Ethics Committee of the Fifth Central Hospital of Tianjin. Periodontal ligament tissues were isolated and were digested with collagenase and trypsin (Gibco) for $60 \mathrm{~min}$. Singlecell suspensions then incubated in growth medium (GM) containing a-modified Eagle's medium (a-MEM) supplemented with $10 \%$ fetal bovine serum (Gibco) and $1 \%$ penicillin/streptomycin in a humidified $5 \%$ $\mathrm{CO}_{2}$ atmosphere at $37^{\circ} \mathrm{C}$.

PDLSCs of the 3 passage were inoculated into 6 -well culture plates at $2 \times 10^{5}$ cells/well. The osteogenic induction medium was standard GM supplemented with $100 \mathrm{nM}$ dexamethasone, $200 \mu \mathrm{M}$ l-ascorbic acid, and $10 \mathrm{mM} \beta$-glycerophosphate (Sigma-Aldrich, USA). Osteogenic differentiation medium was replaced every 3 days.

\section{RNA preparation and quantitative real-time PCR (qRT-PCR)}

Trizol reagent (Life Technologies, USA) and a miRNeasy Mini Kit (Qiagen, Germany) was used to isolate total RNA according to the manufacturer's protocol. For mRNA detection, the first-strand cDNA was the high-capacity cDNA reverse transcription kit (Applied Biosystems, USA) and QuantiTect SYBR Green PCR kit (Qiagen, Germany) were employed. For miRNAs detection, real-time PCR was performed using miDETECT A Track ${ }^{\text {TM }}$ miRNA qRT-PCR Starter Kit (RIBOBIO, China). GAPDH and U6 were used as the normalization for mRNA and miRNA. The primer sequences for the miR-217 and U6(Dai et al., 2019), RUNX2, OPN, OCN, EZH2 and $\beta$-actin(Wang et al., 2016), Wnt1(Zhang et al., 2015) and $\beta$-catenin(Shan et al., 2017) were previously described.

\section{Western blot analysis}

RIPA lysis buffer was used to extract total protein. Equal quantities of proteins were separated by SDSPAGE and transferred onto polyvinylidene difluoride (PVDF) membranes (Millipore). Primary antibodies against RUNX2 (ab23981, Abcam, USA), OCN (ab93876), OPN (ab69498), $\beta$-actin (ab179467), EZH2 (ab195409), Wnt1 (ab15251), $\beta$-catenin (ab16051), and H3K27me3 (ab6002) were selected. After washing, peroxidase-conjugated goat anti-rabbit or goat anti-mouse were used as the secondary 
antibody. The protein blots were visualized using the enhanced chemiluminescence $(E C L)$ luminol reagent (Millipore, USA) and exposed to X-ray film (Fujifilm, Japan). The signal of each target band was normalized to that of the $\beta$-actin band.

\section{Alkaline phosphatase (ALP) assays}

ALP enzyme activity was measured using an alkaline phosphatase detection kit (Biyuntian Biotech Co., China) following the manufacturer's instructions. ALP staining was performed using a NBT/BCIP staining kit (CoWin Biotech, China) following the manufacturer's instructions.

\section{Alizarin Red Staining}

The matrix mineralization was measured by Alizarin Red staining (Sigma, USA) following the manufacturer's instructions. To quantitatively evaluate the mineralized nodules, the staining solution was dissolved in $1 \mathrm{~mL} \mathrm{10 \%} \mathrm{cetylpyridinium} \mathrm{chloride} \mathrm{(Sigma-Aldrich)} \mathrm{for} 1 \mathrm{~h}$ and the absorbance at $570 \mathrm{~nm}$ was detected by spectrophotometric methods.

\section{Cell transfection}

EZH2 overexpression vector, miR-217 mimic, miR-217 inhibitor, miR mimic control (NC-mimic), and miRNA inhibitor control (NC-inhibitor) all purchased from GenePharma (Shanghai, China) and were transfected into PDLSCs using Lipofectamine 3000 reagent (Invitrogen, USA), with all procedures following the manufacturer's protocol.

\section{Dual luciferase reporter gene assay}

PmirGLO vectors (Promega, USA) containing wild-type (wt) or mutation-type (mut) sequences of EZH2 3'untranslated region (3'-UTR) were constructed by GenePharma (Shanghai, China). PmirGLO luciferase reporter gene vector was introduced into PDLSCs cells with co-transfection of miR-217 mimic or mimicNC using Lipofectamine 3000 reagent (Invitrogen). Relative luciferase activity was assessed with Dualluciferase Reporter Assay Kit (Promega) following the manufacturer's protocol.

\section{Chromatin immunoprecipitation assays}

ChIP was carried out using an anti-EZH2 and anti-H3K27me3 antibody (Abcam) and the EZ CHIP KIT (Millipore, USA) following the manufacturer's protocol as described previously(Deng et al., 2018). The results were normalized relative to the input control.

\section{Statistical analysis}

SPSS software (version 16.0; SPSS, Inc., Chicago, IL, USA) was used for statistical analyses. All data are expressed as the mean \pm SD of at least three independent experiments. Student's t test was used to analyze differences between groups. One-way analysis of variance (ANOVA) was employed for multiple group testing. A two-tailed $p<0.05$ was considered statistically significant. 


\section{Results}

\section{MiR-217 was involved in regulating the osteogenic differentiation of PDLSCs}

To investigate the role of miR-217 in regulating osteogenic differentiation, PDLSCs were cultured in osteogenic differentiation medium. The levels of osteoblastic marker genes including RUNX2, OPN and OCN were significantly increased at day 7 and 14 after osteogenic induction (Fig 1A). Consistently, ALP and Alizarin Red staining experiments confirmed the osteoblast phenotype (Fig. 1B-D). Next, we found that miR-217 were significantly increased at day 7 and 14 after osteogenic induction (Fig. 1E). These results indicated that miR-217 might be involved in regulating the osteogenic differentiation of PDLSCs.

\section{MiR-217 promotes the osteogenic differentiation of PDLSCs}

Firstly, PDLSCs were transfected with miR-217 mimic, miR-217 inhibitor and corresponding negative control each three days for total four times during osteogenic differentiation. miR-217 mimic obviously enhanced miR-217 levels and miR-217 inhibitor significantly decreased miR-217 levels (Fig. 2A). miR-217 overexpression apparently promoted osteogenic differentiation, as indicated by the up-regulated osteogenic marker genes RUNX2, OPN and OCN. On the contrary, miR-217 knockdown by miR-217 inhibitor significantly inhibited osteogenic differentiation as indicated by the down-regulated osteogenic marker genes (Fig. 2B). Furthermore, ALP activity and the matrix mineralization levels were significantly promoted after miR-217 mimic treatment and were obviously inhibited after miR-217 inhibitor treatment (Fig. 2C-E). In short, we confirmed the promotion of miR-217 on the osteogenic differentiation of PDLSCs.

\section{MiR-217 promotes the osteogenic differentiation of PDLSCs by targeting EZH2}

In order to further explore the mechanism of miR-217 on osteogenic differentiation of PDLSCs, EZH2 was selected as EZH2 was reported to be a target gene of miR-217 and was closely related to osteogenic differentiation(Xue et al., 2017, Wei et al., 2011). Luciferase reporter assay results suggested that miR-217 could target EZH2 3'-UTR (Fig. 3A). Furthermore, miR-217 overexpression substantially decreased the expression of EZH2 while miR-217 knockdown increased EZH2 mRNA and protein levels (Fig. 3B-C). These results indicated that EZH2 was a direct target of miR-217. Importantly, we found that EZH2 mRNA were significantly decreased at day 7 and 14 after osteogenic induction (Fig. 3D). Next, we explore role of EZH2 in miR-217-regulated osteogenic differentiation. EZH2 overexpression obviously downregulated osteogenic marker genes including RUNX2, OCN and OPN expression. We restored the expression of EZH2 in miR-217-overexpressing PDLSCs by transfecting the cells with EZH2 overexpression plasmids and found that the expression of EZH2 and osteogenic marker genes including RUNX2, OCN and OPN, were inhibited by EZH2 rescue in the miR-217-overexpressing PDLSCs (Fig. 3E). The overexpression of EZH2 also decreased ALP activity and matrix mineralization (Fig. 3F-H). EZH2 overexpression impaired the effect of miR-217 in the osteogenic differentiation of PDLSCs (Fig. 3F-H). Taken together, these results indicated that EZH2 was a functional target of miR-217 on osteogenic differentiation of PDLSCs. 


\section{MiR-217 promotes the osteogenic differentiation ofPDLSCs through EZH2 mediated-inactive of Wnt/ $\beta$ - catenin pathway}

EZH2 was found to inhibit Wnt/ $\beta$-catenin signaling (Wang et al., 2016), which may explain the reason of $\mathrm{EZH} 2$ on osteogenic differentiation to a certain extent. During osteogenesis, Wnt1 and $\beta$-catenin was upregulated while the enrichment of the active H3K4me3 modification was decreased (Fig. 4A-C). EZH2 and H3K27me3 also found to be decreased on the transcription start site of Wnt 1 and $\beta$-catenin (Fig. 4D$E)$. To address these issues, the levels of Wnt 1 genes and $\beta$-catenin were detected in the context of miR217 overexpression or EZH2 overexpression. miR-217 overexpression increased Wnt 1 genes and $\beta$ catenin expression while EZH2 overexpression decreased Wnt 1 genes and $\beta$-catenin expression. Specially, EZH2 overexpression rescued the promotion effect of miR-217 on Wnt/ $\beta$-catenin signaling (Fig. 4F-G). Based on the epigenetic regulation function of EZH2 through methylation on H3K27, we then explored whether EZH2 was involved in the silencing of Wnt-related genes in PDLSCs. Firstly, we conformed that the total H3K27me3 was decreased after miR-217 overexpression while total H3K27me3 was increased after EZH2 overexpression (Fig. 4F-G). Furthermore, ChIP-PCR assay to confirm that EZH2 and H3K27me3 were highly enriched in the promoters of $\beta$-catenin and Wnt1 in osteogenic PDLSCs (Fig. $4 \mathrm{H}-\mathrm{I})$, which indicated that EZH2 was directly bound to Wnt gene promoters. MiR-217 overexpression significantly downregulated the enrichment of EZH2 and H3K27me3 in the promoters of $\beta$-catenin and Wnt1 in osteogenic PDLSCs. However, EZH2 overexpression significantly upregulated the enrichment of $\mathrm{EZH} 2$ and H3K27me3 in the promoters of $\beta$-catenin and Wnt1 in osteogenic PDLSCs (Fig. 4H-I). Our results indicated that miR-217 promoted the osteogenic differentiation of PDLSCs through downregulating EZH2 expression and eliminating the inhibition function on transcription of Wnt 1 and $\beta$ catenin.

\section{Discussion}

PDLSCs with multilineage differentiation, are considered key candidates for bone regenerative applications(Tassi et al., 2017). Therefore, it is essential to understand the mechanisms on PDLSCs multilineage differentiation potential. In this study, miR-217 was demonstrated to promote the osteogenic differentiation of PDLSCs. Similarly, miR-217 was reported to promote cell proliferation and osteogenic differentiation of bone marrow-derived mesenchymal stem cells by inhibiting DKK1 during the development of steroid-associated osteonecrosis(Dai et al., 2019). Gu et al. indicated that miR-217 was upregulated in the process of 1,25-dihydroxyvitamin D3-induced osteogenic differentiation of human adipose-derived Mesenchymal stem cells(Gu et al., 2017). In our study, we indicated that miR-217 promoted PDLSCs osteogenesis by targeting EZH2 and activating Wnt/ $\beta$-catenin axis.

The epigenetic modifier enhancer of zeste homolog 2 (EZH2) was a subunit of polycomb repressor complex 2 (PRC2) and was a histone three lysine 27 (H3K27) methyltransferase(Sparmann and van Lohuizen, 2006). EZH2 is required for bone formation and anterior/posterior skeletal patterning in mice during development(Wyngaarden et al., 2011, Schwarz et al., 2014). EZH2 was found to inhibit osteogenic differentiation potential in vitro and in vivo(Hemming et al., 2014). Inhibition of EZH2 
enzymatic activity and knockdown of EZH2 gene expression promoted osteogenic differentiation by MSC(Chen et al., 2016). Chromatin immunoprecipitation analysis has revealed that the presence of EZH2 and its H3K27me3-associated modification is reduced at the transcription start site (TSS) of key osteogenic transcription factor RUNX2 during osteogenic differentiation(Wei et al., 2011, Hemming et al., 2014). ZBTB16, MX1, and FHL1 was identified to be EZH2 targets regulating osteogenic differentiation in mesenchymal stem cells(Hemming et al., 2016). In our study, EZH2 and its H3K27me3-associated modification was found to be present along the transcription start site of Wnt1 and $\beta$-catenin promoters. During osteogenesis, Wnt1 and $\beta$-catenin was upregulated coinciding with the disappearance of EZH2 and H3K27me3 on the transcription start site of these genes and the enrichment of the active H3K4me3 modification. Overexpression of EZH2 downregulated the transcript levels and protein levels of Wnt1 and $\beta$-catenin during osteogenesis.

In summary, our findings indicate that miR-217 and EZH2 play an important role in the osteogenesis of PDLSCs. The decrease of EZH2 and H3K27me3 activated Wnt1/ $\beta$-catenin signaling pathway. These results help to determine the possible mechanism of miR-217 and EZH2 in regulating PDLSCs differentiation. Our results provide new insight indicating that miR-217 possesses great potential as a novel class of therapeutic targets for bone regeneration.

\section{Declarations}

\section{Acknowledgements}

None.

\section{Author's contributions}

H.J. and PZ.J. have given substantial contributions to the conception and the design of the manuscript, H.J. and PZ.J. to acquisition, analysis and interpretation of the data. All authors have participated to drafting the manuscript, H.J. revised it critically. All authors read and approved the final version of the manuscript.

\section{Funding}

Not applicable.

\section{Conflict of Interest}

The authors declare that they have no competing interests.

\section{Ethics approval}

All procedures performed in studies involving human participants were in accordance with the ethical standards of the institutional and national research committee and with the 1964 Helsinki Declaration and its later amendments or comparable ethical standards. 


\section{Consent to participate}

The study protocol was approved by the Fifth Central Hospital of Tianjin on human research.

\section{Consent for publication}

All patients have provided written informed consent for the publication of any associated data and accompanying images.

\section{Availability of data and material}

All data generated and analyzed during this study are included in this published article.

\section{References}

1. Joshi D, Garg T, Goyal AK, Rath G. Advanced drug delivery approaches against periodontitis. Drug Deliv. 2016;23(2):363-77.

2. Son $\mathrm{H}$, Jeon M, Choi HJ, Lee HS, Kim IH, Kang CM, et al. Decellularized human periodontal ligament for periodontium regeneration. PLoS One. 2019;14(8):e0221236.

3. Nagata M, Iwasaki K, Akazawa K, Komaki M, Yokoyama N, Izumi Y, et al. Conditioned Medium from Periodontal Ligament Stem Cells Enhances Periodontal Regeneration. Tissue Eng Part A. 2017;23(910):367-77.

4. Menicanin D, Mrozik KM, Wada N, Marino V, Shi S, Bartold PM, et al. Periodontal-ligament-derived stem cells exhibit the capacity for long-term survival, self-renewal, and regeneration of multiple tissue types in vivo. Stem Cells Dev. 2014;23(9):1001-11.

5. Xu Y, Ren C, Zhao X, Wang W, Zhang N. microRNA-132 inhibits osteogenic differentiation of periodontal ligament stem cells via GDF5 and the NF-kappaB signaling pathway. Pathol Res Pract. 2019;215(12):152722.

6. Eskildsen T, Taipaleenmaki H, Stenvang J, Abdallah BM, Ditzel N, Nossent AY, et al. MicroRNA-138 regulates osteogenic differentiation of human stromal (mesenchymal) stem cells in vivo. Proc Natl Acad Sci U S A. 2011;108(15):6139-44.

7. Zhang Y, Li S, Yuan S, Zhang H, Liu J. MicroRNA-23a inhibits osteogenesis of periodontal mesenchymal stem cells by targeting bone morphogenetic protein signaling. Arch Oral Biol. 2019;102:93-100.

8. Schmalz G, Li S, Burkhardt R, Rinke S, Krause F, Haak R, et al. MicroRNAs as Salivary Markers for Periodontal Diseases: A New Diagnostic Approach? Biomed Res Int. 2016;2016:1027525.

9. Nie X, Fan J, Li H, Yin Z, Zhao Y, Dai B, et al. miR-217 Promotes Cardiac Hypertrophy and Dysfunction by Targeting PTEN. Mol Ther Nucleic Acids. 2018;12:254-66.

10. Li J, Liu B, Xue H, Zhou QQ, Peng L. miR-217 Is a Useful Diagnostic Biomarker and Regulates Human Podocyte Cells Apoptosis via Targeting TNFSF11 in Membranous Nephropathy. Biomed Res Int. 2017;2017:2168767. 
11. Jiang W, Wang Q, Yu X, Lu T, Zhang P. MicroRNA-217 relieved neuropathic pain through targeting tolllike receptor 5 expression. J Cell Biochem. 2019;120(3):3009-17.

12. Zhu Y, Zhao H, Feng L, Xu S. MicroRNA-217 inhibits cell proliferation and invasion by targeting Runx2 in human glioma. Am J Transl Res. 2016;8(3):1482-91.

13. Nishioka C, Ikezoe T, Yang J, Nobumoto A, Tsuda M, Yokoyama A. Downregulation of miR-217 correlates with resistance of $\mathrm{Ph}(+)$ leukemia cells to $\mathrm{ABL}$ tyrosine kinase inhibitors. Cancer Sci. 2014;105(3):297-307.

14. Zhao WG, Yu SN, Lu ZH, Ma YH, Gu YM, Chen J. The miR-217 microRNA functions as a potential tumor suppressor in pancreatic ductal adenocarcinoma by targeting KRAS. Carcinogenesis. 2010;31(10):1726-33.

15. Dai Z, Jin Y, Zheng J, Liu K, Zhao J, Zhang S, et al. MiR-217 promotes cell proliferation and osteogenic differentiation of BMSCs by targeting DKK1 in steroid-associated osteonecrosis. Biomed Pharmacother. 2019;109:1112-9.

16. Wang H, Meng Y, Cui Q, Qin F, Yang H, Chen Y, et al. MiR-101 Targets the EZH2/Wnt/beta-Catenin the Pathway to Promote the Osteogenic Differentiation of Human Bone Marrow-Derived Mesenchymal Stem Cells. Sci Rep. 2016;6:36988.

17. Zhang JG, Shi Y, Hong DF, Song M, Huang D, Wang CY, et al. MiR-148b suppresses cell proliferation and invasion in hepatocellular carcinoma by targeting WNT1/beta-catenin pathway. Sci Rep. 2015;5:8087.

18. Shan L, Li Y, Jiang H, Tao Y, Qian Z, Li L, et al. Huaier Restrains Proliferative and Migratory Potential of Hepatocellular Carcinoma Cells Partially Through Decreased Yes-Associated Protein 1. J Cancer. 2017;8(19):4087-97.

19. Deng L, Hong H, Zhang X, Chen D, Chen Z, Ling J, et al. Down-regulated IncRNA MEG3 promotes osteogenic differentiation of human dental follicle stem cells by epigenetically regulating Wnt pathway. Biochem Biophys Res Commun. 2018;503(3):2061-7.

20. Xue J, Liu Y, Luo F, Lu X, Xu H, Liu X, et al. Circ100284, via miR-217 regulation of EZH2, is involved in the arsenite-accelerated cell cycle of human keratinocytes in carcinogenesis. Biochim Biophys Acta Mol Basis Dis. 2017;1863(3):753-63.

21. Wei Y, Chen YH, Li LY, Lang J, Yeh SP, Shi B, et al. CDK1-dependent phosphorylation of EZH2 suppresses methylation of $\mathrm{H} 3 \mathrm{~K} 27$ and promotes osteogenic differentiation of human mesenchymal stem cells. Nat Cell Biol. 2011;13(1):87-94.

22. Tassi SA, Sergio NZ, Misawa MYO, Villar CC. Efficacy of stem cells on periodontal regeneration: Systematic review of pre-clinical studies. J Periodontal Res. 2017;52(5):793-812.

23. Gu H, Xu J, Huang Z, Wu L, Zhou K, Zhang Y, et al. Identification and differential expression of microRNAs in 1,25-dihydroxyvitamin D3-induced osteogenic differentiation of human adiposederived mesenchymal stem cells. Am J Transl Res. 2017;9(11):4856-71.

24. Sparmann A, van Lohuizen M. Polycomb silencers control cell fate, development and cancer. Nat Rev Cancer. 2006;6(11):846-56. 
25. Wyngaarden LA, Delgado-Olguin P, Su IH, Bruneau BG, Hopyan S. Ezh2 regulates anteroposterior axis specification and proximodistal axis elongation in the developing limb. Development. 2011;138(17):3759-67.

26. Schwarz D, Varum S, Zemke M, Scholer A, Baggiolini A, Draganova K, et al. Ezh2 is required for neural crest-derived cartilage and bone formation. Development. 2014;141(4):867-77.

27. Hemming S, Cakouros D, Isenmann S, Cooper L, Menicanin D, Zannettino A, et al. EZH2 and KDM6A act as an epigenetic switch to regulate mesenchymal stem cell lineage specification. Stem Cells. 2014;32(3):802-15.

28. Chen YH, Chung CC, Liu YC, Yeh SP, Hsu JL, Hung MC, et al. Enhancer of Zeste Homolog 2 and Histone Deacetylase 9c Regulate Age-Dependent Mesenchymal Stem Cell Differentiation into Osteoblasts and Adipocytes. Stem Cells. 2016;34(8):2183-93.

29. Hemming S, Cakouros D, Vandyke K, Davis MJ, Zannettino AC, Gronthos S. Identification of Novel EZH2 Targets Regulating Osteogenic Differentiation in Mesenchymal Stem Cells. Stem Cells Dev. 2016;25(12):909-21.

\section{Figures}
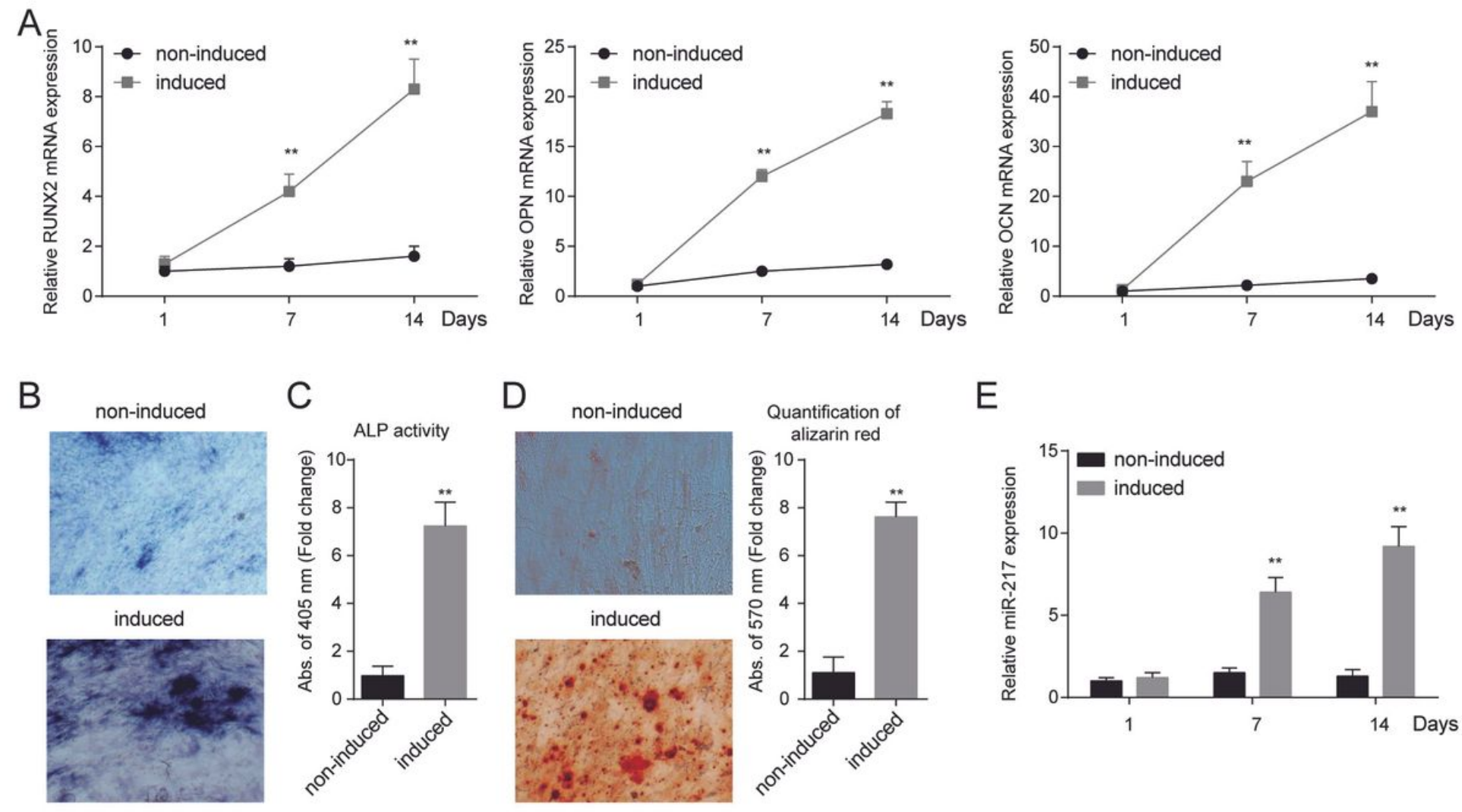

$E$

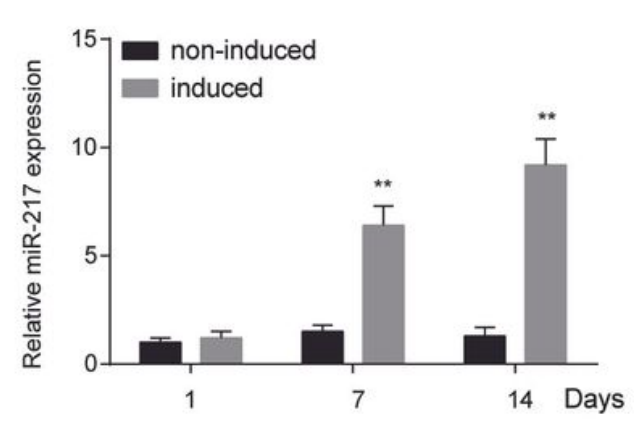

Figure 1 
miR-217 is upregulated during the osteogenic differentiation of PDLSCs. (A) The mRNA levels of the osteoblastic marker genes RUNX2, OPN and OCN were analyzed by qRT-PCR at day 1, 7 and 14, with GAPDH as a control. (B) ALP staining was measured at day 14. (C) ALP activity was measured at day 14. (D) Alizarin Red staining was measured at day 14. (E) The miR-27 levels were analyzed by qRT-PCR at day 1, 7 and 14 after osteogenic induction, with U6 as a control. ${ }^{*} p<0.01$ versus the non-induced group.
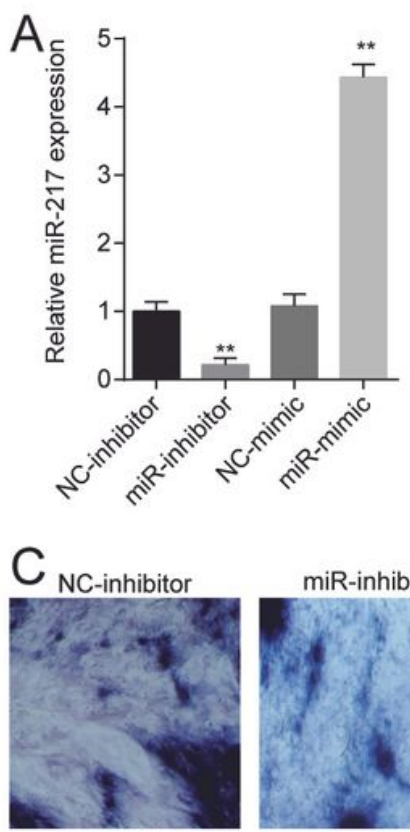

NC-mimic

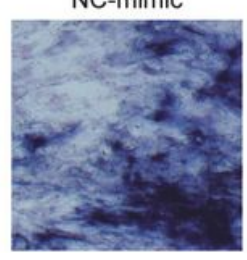

B

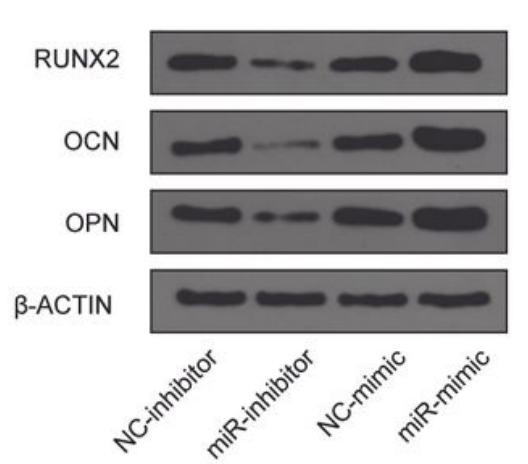

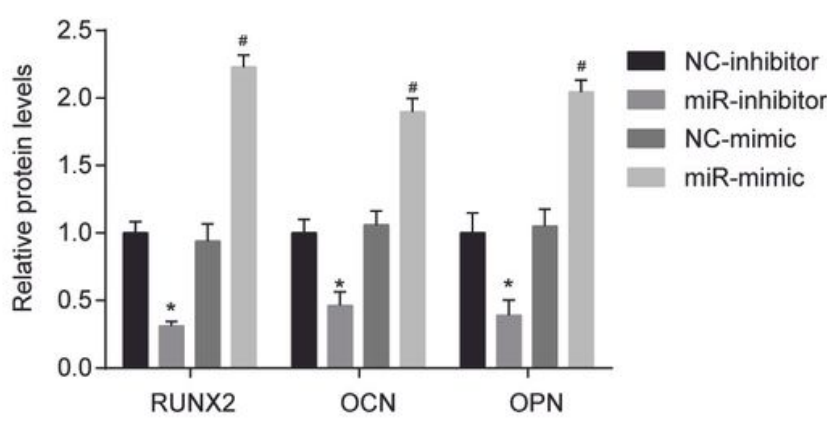

D

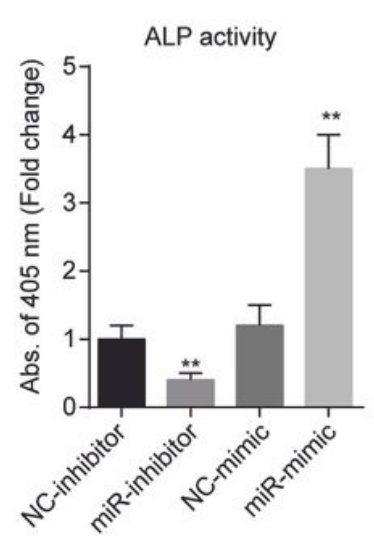

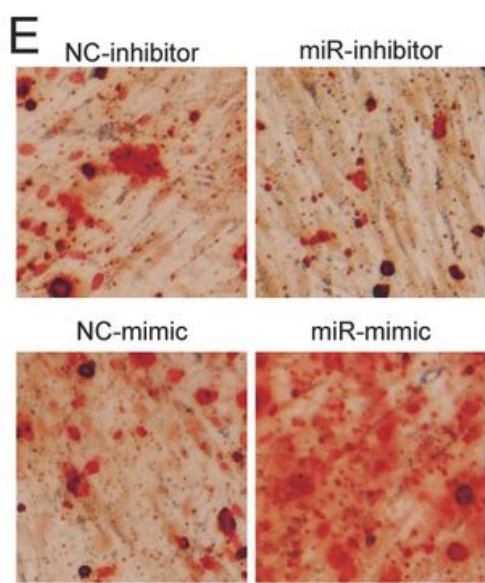

Quantification of alizarin red

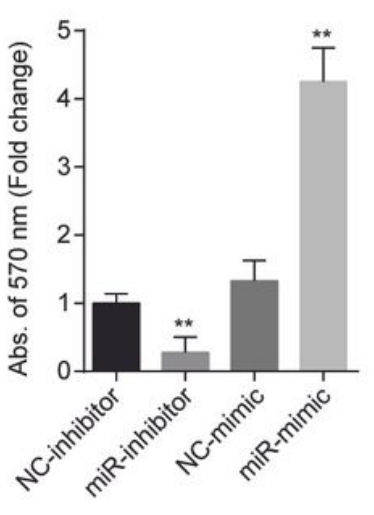

\section{Figure 2}

miR-217 promotes the osteogenic differentiation of PDLSCs. During osteogenic differentiation, PDLSCs were transfect miR-217 mimic, miR-217 inhibitor and corresponding negative control each three days for total four times. (A) miR-217 levels in PDLSCs. (B) The osteoblastic marker genes RUNX2, OPN and OCN were analyzed by western blot with $\beta$-actin as a control. (C) ALP staining was measured at day 14 . (D) ALP activity was measured at day 14 . (E) Alizarin Red staining was measured at day 14 and quantification was shown at right. ${ }^{*} p<0.05,{ }^{\star *} p<0.01$ versus NC-inhibitor; $\# p<0.05$, $\# \# p<0.01$ versus NC-mimic. 
A

WT EZH2 3'UTR: 5' auuuagaaaaagaacAUGCAGUu 3' miR-217 : $3^{\prime}$ agguuagucaaggacUACGUCAu $5^{\prime}$ MUT EZH2 3'UTR: 5' auuuagaaaaagaacACGUACUu 3'

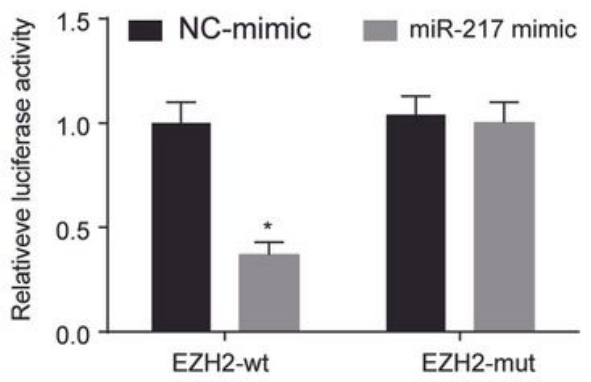

D

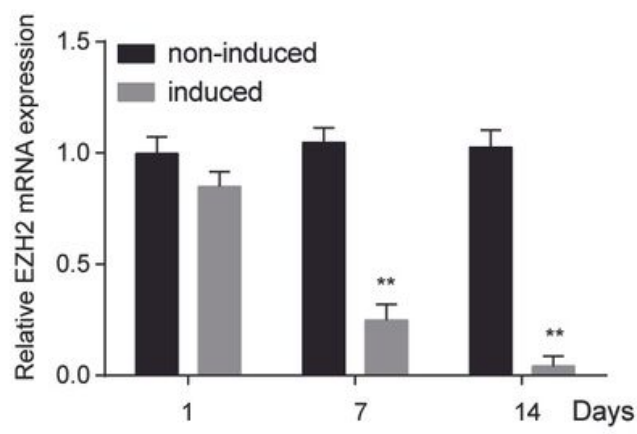

B

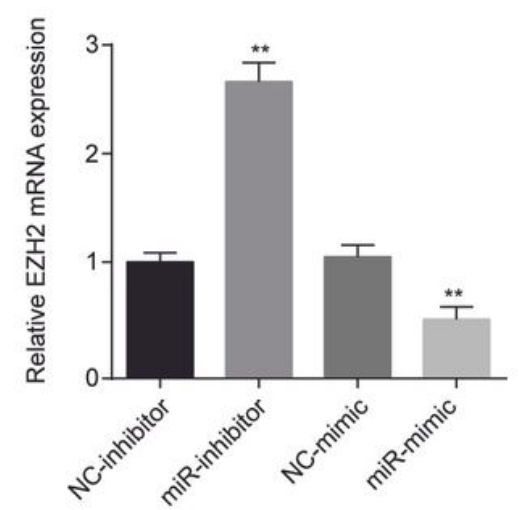

E

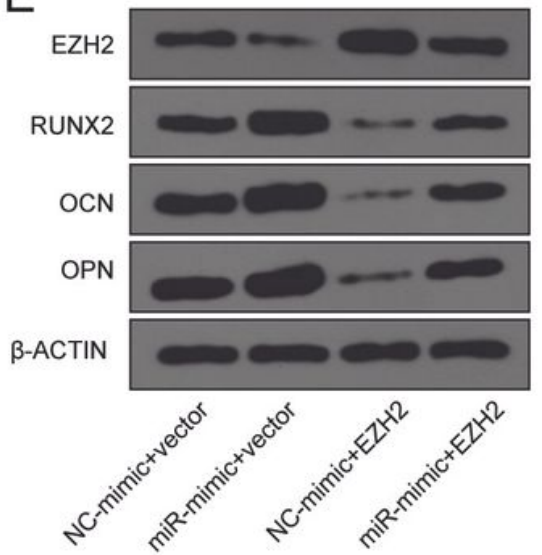

C
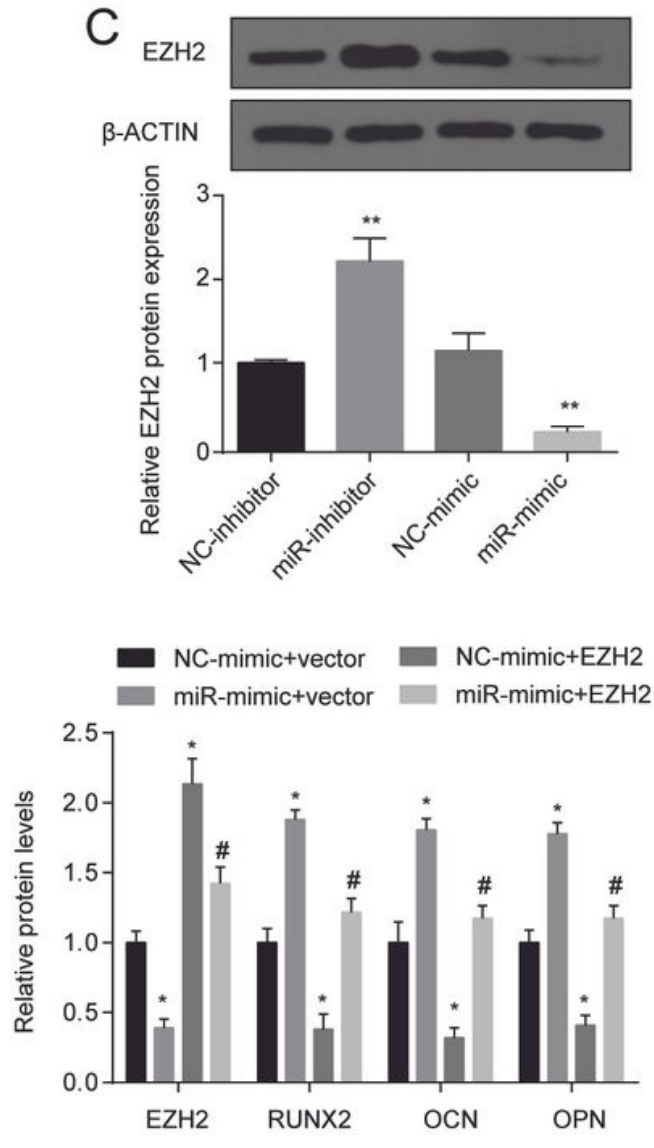

$\mathrm{F}$

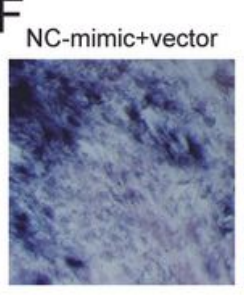

$\mathrm{NC}-$ mimic $+\mathrm{EZH} 2$

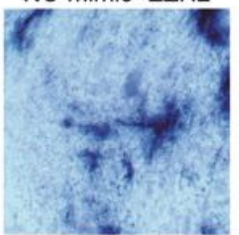

G

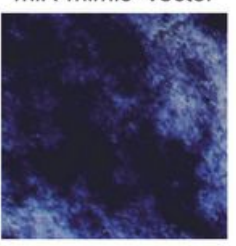

miR-mimic+EZH2

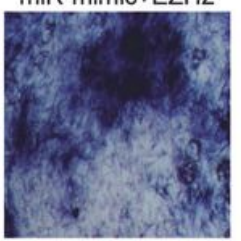

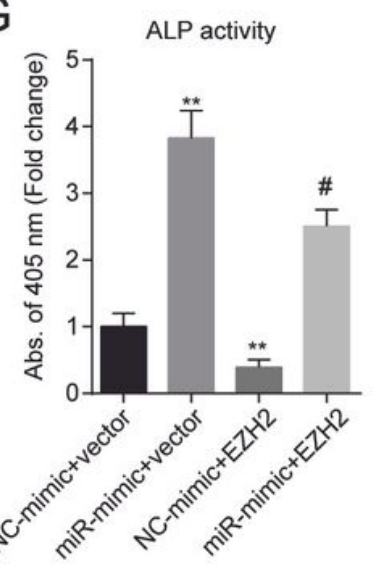

$\mathrm{H}_{\text {NC-mimictvector }}$

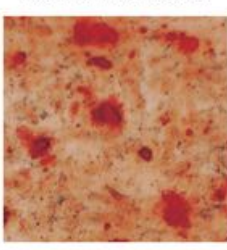

$\mathrm{NC}-$ mimic $+\mathrm{EZH} 2$

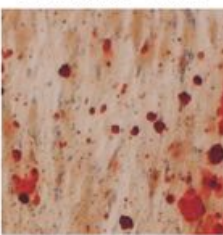

Quantification of alizarin red

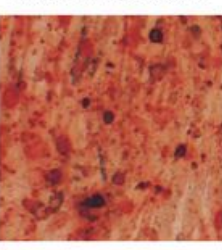

miR-mimic+EZH2

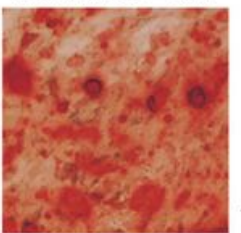

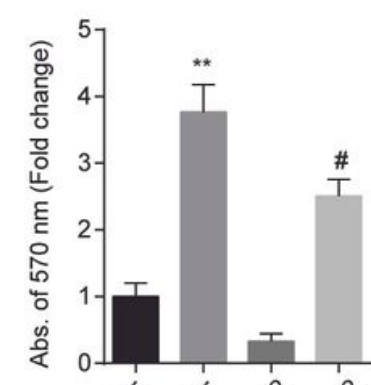

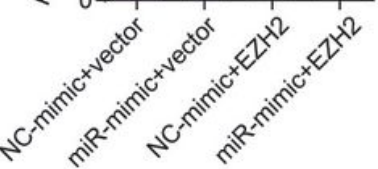

\section{Figure 3}

miR-217 promotes the osteogenic differentiation of PDLSCs targeting EZH2. During osteogenic differentiation, PDLSCs were transfect with miR-217 mimic or EZH2 overexpression plasmids each three days for total four times. (A) Schematic representation of the miR-217 site in the EZH2 3'-UTR. Luciferase assays were performed to confirm the target relationship between miR-217 and EZH2 in PDLSCs. Firefly luciferase activity was standardized to a Renilla luciferase control. (B) EZH2 mRNA expression in PDLSCs. (C) EZH2 protein expression in PDLSCs. (D) EZH2 mRNA expression at day 1, 7 and 14 after osteogenic induction. (E) EZH2, RUNX2, OPN and OCN protein expression in PDLSCs. (F) ALP staining was measured at day 14 after indicated treatment. (G) ALP activity was measured at day 14 after 
indicated treatment. $(\mathrm{H})$ Alizarin Red staining was measured at day 14 after indicated treatment and quantification was shown at right. ${ }^{*} p<0.05,{ }^{* \star} p<0.01$ versus NC-mimic+vector; $\# p<0.05, \# \#<0.01$ versus miR-mimic+vector.
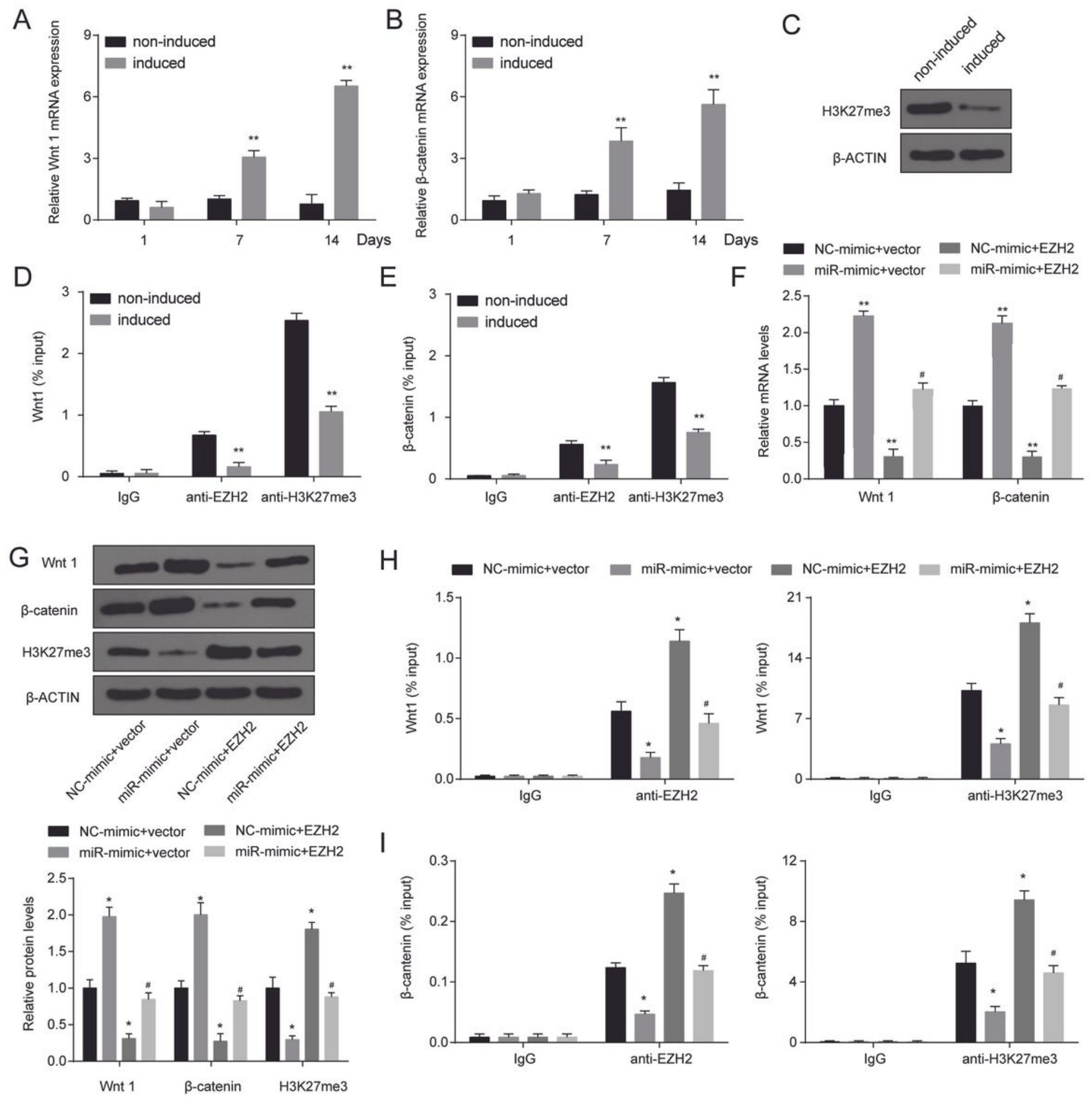

\section{Figure 4}

Downregulated EZH2 activated the Wnt signaling pathway via the process of H3K27 trimethylation. During osteogenic differentiation, PDLSCs were transfect with miR-217 mimic or EZH2 overexpression plasmids each three days for total four times. (A) The mRNA levels of Wnt1 were analyzed by qRT-PCR at 
day 1,7 and 14, with GAPDH as a control. (B) The mRNA levels of $\beta$-catenin were analyzed by qRT-PCR at day 1, 7 and 14, with GAPDH as a control. (C) The protein levels of H3K27me3 was analyzed at day 14, with GAPDH as a control. (D) ChIP assays showed the enrichment of EZH2 and H3K27me3 to Wnt 1 promoters in PDLSCs with or without osteogenic induction. (E) ChIP assays showed the enrichment of EZH2 and H3K27me3 to $\beta$-catenin promoters in PDLSCs with or without osteogenic induction. $(F)$ The expression of Wnt 1 and $\beta$-catenin were evaluated by qRT-PCR with osteogenic induction for $14 \mathrm{~d}$. (G) The expression of Wnt 1 and $\beta$-catenin were evaluated by western blot analysis with osteogenic induction for $14 \mathrm{~d}$. $(\mathrm{H})$ ChIP assays showed the enrichment of EZH2 and IgG to Wnt 1 and $\beta$-catenin promoters in PDLSCs. (I) Enrichment of H3K27me3 in promoters of Wnt 1 and $\beta$-catenin promoters in PDLSCs. * $p<$ $0.05,{ }^{* \star} p<0.01$ versus NC-mimic+vector; $\# p<0.05, \# \# p<0.01$ versus miR-mimic+vector. 